\title{
ON RELIABLE FINITE ELEMENT METHODS FOR EXTREME LOADING CONDITIONS
}

\author{
K.-J. Bathe (kjb@mit.edu) \\ Massachusetts Institute of Technology, Cambridge, MA 02139, \\ U.S.A.
}

\begin{abstract}
In this paper we focus on the analysis of solids and structures when these are subjected to extreme conditions of loading resulting in large deformations and possibly failure. The analysis should be conducted with finite element methods that are as reliable as possible and effective. The requirement of reliability is important in any finite element analysis but is particularly important in simulations involving extreme loadings since physical test data are frequently not available, or only available for some similar conditions. To then reach a high level of confidence in the computed solutions requires that reliable finite element procedures be used.

While in this paper a large field of analysis is covered, the presentation is narrow because it only focuses on our research results, mostly published in the last decade (since 1995), and only on some of our contributions. Hence, this paper is not written to fully survey the field.
\end{abstract}

Key words: finite element methods, structures, fluids, extreme loading

\section{Introduction}

Finite element methods are now widely used in engineering analysis and we can expect a continued growth in the use of these methods. Finite element programs are extensively employed for linear and nonlinear analyses, and the simulations of highly nonlinear events are of much interest ${ }^{1-3}$. In particular, the simulations may be used to postulate accidents and natural disasters, and thus can be used to study how a structure will perform in such severe events. Then, if necessary, remedies in the design of a structure can be undertaken, and equally important, warning devices might be installed, that all lead to greater public safety.

An important point is that for large civil and mechanical engineering structures (long-span bridges, off-shore plants, storage tanks, power plants, chemical plants, underground storage caverns, to name just a few), physical tests can only be performed to a limited extent. Parts of the structures can 
be tested (like connections between pipes) but a complete structure may only be tested when completely assembled in the field and sometimes that is not possible either. Hence, the simulations of such structures subjected to severe loadings probe into, and try to predict, the future based on scarce actual physical tests. It is then very important to use reliable finite element methods in order to have the highest possible confidence in the computed results ${ }^{3}$.

The objective in this paper is to survey some finite element analysis techniques with a particular focus on the reliability of the methods. Of course, any simulation starts with the selection of a mathematical model, and this model must be chosen judiciously. Once an appropriate mathematical model has been selected, for the questions asked, the finite element analysis is performed.

In this paper, we consider the solution of problems involving 2D and 3D solids, plates and shells, fluid-structure interactions, and general multiphysics events. The simulation of structures subjected to extreme loading conditions frequently comprises multi-physics events that involve fluids and severe thermal effects. For all these analyses, we need to use effective and reliable finite elements, efficient methods for the solution of the equations, for steady-state (static) and transient conditions, and finally, as far as possible, appropriate error measures.

While a large analysis field is covered in this paper, we shall focus only on our research accomplishments since 1995, and that also only partially. Indeed, throughout our research endeavors since the 1970's, the aim was always to only develop reliable and effective finite element procedures ${ }^{3}$.

Therefore, this paper is not intended to be a survey paper, neither to give all of our achievements nor to give adequate acknowledgement of the many important works of other researchers. All we focus on is to survey some valuable analysis methods for structures in extreme loading conditions.

\section{On the Selection of the Mathematical Model}

The first step of any structural analysis involves the selection of an appropriate mathematical model ${ }^{3,4}$. This model needs to be selected based on the geometry, material properties, the loading and boundary conditions (the effect of the 'rest of the universe' on the structure) and, most importantly, the questions asked by the analyst. If fluid-structure interactions are important, then the mathematical model should also include the fluid and the loading and boundary conditions thereon.

The purpose of the analysis is of course to answer certain questions regarding the stiffness, strength and possible failure of the structure under 
consideration. In the case of extreme loading conditions, we would like to ascertain the behavior of the structure in postulated accidents, either manmade or imposed by nature, or in natural disasters. Hence, when studying the behavior of the structure, we would like to predict the future not only when the structure is operating in normal conditions, which requires a linear analysis, but also when the structure is subjected to extreme conditions of loading, which requires a highly nonlinear analysis.

The mathematical model should naturally be as simple as possible to answer the engineering questions but not be too simple (an observation attributed to A. Einstein) because the answers may then be erroneous and misleading. For most structures, it is necessary to choose a mathematical model that can only be solved using numerical methods and finite element procedures are widely used.

It is clear that the finite element solution of the mathematical model will contain all the assumptions of the mathematical model and hence cannot predict any response not contained in this model. Selecting the appropriate mathematical model is therefore most important. But it is also clear that the analysis can only give insight into the physical behavior of a structure, that is, nature, because it is impossible to reproduce nature exactly.

In this paper we only consider deterministic analyses. If nondeterministic simulations need to be carried out, then still, the considerations given here are all valid because the deterministic procedures are basic methods used in those analyses as well ${ }^{1}$.

A fundamental question must always be whether the mathematical model used is appropriate. This question can be addressed by the process of hierarchical modeling ${ }^{3-4}$. In this process of mathematical modeling and finite element solution, it is best to use one finite element program for the solution of the different linear and nonlinear mathematical models, because the assumptions in the finite element procedures are then the same in all solutions. In the case of extreme conditions of loading, this finite element program need be used for linear and highly nonlinear structural and multiphysics problems, including fluid flows, severe temperature conditions, and the full mechanical interactions. In this paper we are focusing on ADINA for such analyses.

\section{The Finite Element Solution of Solids and Structures}

With the mathematical model chosen, the finite element procedures are used to solve the model. It is important that in this phase of the analysis well-founded and reliable procedures be used. By reliability of a finite element procedure we mean that in the solution of a well-posed mathematical model, the procedures always, for a reasonable finite element 
mesh, give a reasonable solution. And if the mesh is reasonably fine, an accurate solution of the chosen mathematical model is obtained ${ }^{3}$.

It is sometimes argued that since the geometry, material conditions, loadings are not known to great accuracy, there is no need to solve the mathematical model accurately. This is a reasonable argument provided the mathematical model is solved to 'sufficient' accuracy and a control on the level of accuracy is available. Such a control on the accuracy of the finite element solution of the mathematical model is difficult to achieve and reliable finite element procedures are best used.

The reliability of a finite element procedure means in particular that when some geometric or material properties are changed in the mathematical model, then for a given finite element mesh the accuracy of the finite element solution does not drastically decrease. Hence, pure displacement-based finite element methods are not reliable when considering almost incompressible materials (like a rubber material, or a steel in large strains). As well-known, these methods 'lock', and for such analyses, well-founded mixed methods need be used ${ }^{3}$.

To exemplify what can go wrong in a finite element solution when unreliable finite element procedures are used, we refer to ref. 3, page 474, where the computed frequencies of a cantilever bracket are given. If reduced integration is used, then phantom frequencies (that are totally nonphysical) are predicted. Such ghost frequencies may also be predicted when some hour-glass control with reduced integration is employed.

Similar situations also arise in the analysis of plates and shells, when reduced integration is employed, but in shell analyses, in addition, the use of flat shell elements can result in unreliable solutions, see Section 3.2. And similar conditions can also arise in the analysis of fluids and the interactions with structures, see Section 4.

\subsection{SOLIDS}

The use of well-formulated mixed methods has greatly enhanced the reliable analysis of solids and structures ${ }^{3}$.

Considering the analysis of solids, in large strains, the condition of an almost incompressible material response is frequently reached. This situation is of course encountered in simulations involving rubber-like materials (that already are almost incompressible in small strain conditions) but also in simulations of many inelastic conditions, like elasto-plasticity and visco-plasticity. In these analyses, the displacement/pressure (u/p) finite elements are very effective and if the appropriate pressure interpolations are chosen, the elements are also optimal ${ }^{3}$. An optimal element uses for a given 
displacement interpolation the highest pressure interpolation that satisfies the inf-sup condition ${ }^{3-6}$

$$
\inf _{q_{h} \in Q_{h}} \sup _{\mathbf{v}_{h} \in V_{h}} \frac{\left(q_{h}, \operatorname{div} \mathbf{v}_{h}\right)}{\left\|q_{h}\right\|_{0}\left\|\mathbf{v}_{h}\right\|_{1}} \geq \beta>0
$$

where $V_{h}$ is the finite element displacement space, $Q_{h}$ is the finite element pressure space, and $\beta$ is a constant independent of $h$ (the element size).

Reference 3 gives a table of $\mathrm{u} / \mathrm{p}$ elements that satisfy the above inf-sup condition. These elements can of course also be used for analyses that do not involve the incompressibility condition (but are computationally slightly more costly than the pure displacement-based elements).

For 2D solutions, for example, the 9/3 element ( 9 nodes for the displacement interpolations, and 3 degrees of freedom for the pressure interpolation) is an effective element. But, in practice, a 4-node element is desirable and frequently the 4/1 element (4 nodes for the displacement interpolations and a constant pressure) is used. While the $4 / 1$ element does not satisfy the above inf-sup condition, it can be quite effective when used with care. A 4-node element that does satisfy the inf-sup condition was presented by Pantuso and Bathe $\mathrm{e}^{7,8}$. Of course, equivalent elements exist for 3D solutions.

In any simulation, it is desirable to obtain, as the last step of the analysis, some indication regarding the accuracy of the finite element solution when measured on the exact solution of the mathematical model. We briefly address this issue in Section 3.4.

\subsection{PLATES AND SHELLS}

In the analysis of plates and shells, the situation is more complex than encountered in the analysis of solids. Here, for a given mesh, an optimal element would give the same error irrespective of the thickness of the plate or shell, and for any plate and shell geometry, boundary conditions and admissible loading used ${ }^{3,5}$. As well known, all displacement-based shell elements formulated using the Reissner-Mindlin kinematic assumption do not satisfy this condition in the analysis of general shells, and for this reason research has focused on the development of mixed elements, based in essence on the general formulation:

Find $\boldsymbol{u}_{h} \in V_{h}$ and $\xi_{h} \in E_{h}$

$$
\begin{gathered}
a\left(\mathbf{u}_{h}, \mathbf{v}_{h}\right)+b\left(\boldsymbol{\xi}_{h}, \mathbf{v}_{h}\right)=f\left(\mathbf{v}_{h}\right) \quad \forall \mathbf{v}_{h} \in V_{h} \\
b\left(\boldsymbol{\eta}_{h}, \mathbf{u}_{h}\right)-t^{2} c\left(\boldsymbol{\eta}_{h}, \boldsymbol{\xi}_{h}\right)=\mathbf{0} \quad \forall \boldsymbol{\eta}_{h} \in E_{h}
\end{gathered}
$$


where $a(\cdot, \cdot), b(\cdot, \cdot)$, and $c(\uparrow, \square)$, are bilinear forms, $f(\cdot)$ is a linear form, $t$ is the thickness of the shell, and $V_{h}, E_{h}$ are the finite element displacement and strain spaces.

Mixed elements, however, then should satisfy the consistency condition, the ellipticity condition, and ideally the inf-sup condition ${ }^{5,6,9-12}$

$$
\sup _{\mathbf{v}_{h} \in V_{h}} \frac{b\left(\boldsymbol{\eta}_{h}, \mathbf{v}_{h}\right)}{\left\|\mathbf{v}_{h}\right\|_{V}} \geq c \sup _{\mathbf{v} \in V} \frac{b\left(\boldsymbol{\eta}_{h}, \mathbf{v}\right)}{\|\mathbf{v}\|_{V}} \quad \forall \boldsymbol{\eta}_{h} \in E_{h}
$$

where $V$ is the complete (continuous) displacement space, and $c$ is a constant independent of $t$ and $h$.

If an element satisfies these conditions, the discretization is optimal. However, the first mixed elements proposed were not tested for these conditions, and only relatively lately a more rigorous testing has been proposed $^{13,14}$.

Of course, the resulting test problems can (and should) also be used to evaluate any already earlier-proposed shell element, even if it seems that the element is effective. For example, when so evaluating flat shell elements formulated by superimposing membrane and bending actions, a non-convergence behavior may be found, see ref. 12 .

We have proposed some time ago the MITC plate and shell elements, see refs. 3, 15 and the references therein, and have also given some further recent MITC developments for shells ${ }^{16,17}$. For the analysis of plates, the infsup condition can be re-written into a simpler form and we were able to check that the MITC plate elements are optimal (or close thereto) ${ }^{9,10}$ (see Section 7.1).

However, for shell elements the inf-sup condition needs to be evaluated as given above and in general only numerical tests seem possible because $V$ is the complete space in which the shell problem is posed ${ }^{11,12}$. Then it can be equally effective to instead solve well-designed test problems ${ }^{12,13,18-20}$. In these tests an appropriate norm to measure the error must be used - a norm that ideally is applicable to all shell problems - and the s-norm proposed by Hiller and Bathe is effective ${ }^{12,20}$.

The difficulty in formulating a general shell element lies in that the element should ideally be optimal in the analysis of membrane-dominated shells, bending-dominated shells, and mixed- behavior shells. For the membrane-dominated case, actually, the displacement-based shell elements perform satisfactorily but for the bending-dominated and mixed cases, the displacement-based elements 'lock' and a mixed formulation need be used - which satisfies consistency, ellipticity, and ideally the inf-sup condition. The inf-sup condition can be by-passed, but then nonphysical numerical factors enter the element formulation. 
At present, there seems no shell element formulated based on the Reissner-Mindlin assumption that is 'proven analytically' to satisfy all three conditions. However, well-designed numerical tests can be performed to identify whether the conditions seem to be satisfied and the MITC shell elements have performed well in such tests. Indeed, the MITC4 shell element (the 4-node element ${ }^{3}$ ) has shown excellent convergence and is used abundantly in a number of finite element codes (see Section 7.2).

Considering convergence studies of the general shell elements used in engineering practice, a basic step was to identify the underlying shell mathematical model used ${ }^{21}$. The different terms in the variational formulation of this shell model can thus also be studied, and it is possible to identify how well the specific terms are approximated in the finite element solution $^{22}$.

The MITC interpolations can of course also be employed in the formulation of 3D-shell elements and here too the underlying shell model was identified ${ }^{23}$.

\subsection{NONLINEAR ANALYSIS OF SOLIDS AND STRUCTURES, INCLUDING CONTACT CONDITIONS}

The above considerations naturally also hold for geometric and material nonlinear analyses. The large deformation analysis of solids and structures has now been firmly established ${ }^{3,24}$, although of course improvements are sought, specifically in establishing more comprehensive material models. A particular area of interest is to increase the accuracy of the response predictions when considering inelastic orthotropic metals, where the anisotropy may exist initially or be induced by the response ${ }^{25-27}$. The elastic response may be anisotropic, the yielding may be anisotropic and the directions and magnitude of anisotropy may change during the response.

The development of increasingly more comprehensive material models will clearly continue for some time and will also involve the molecular modeling of materials and coupling of these models to finite element discretizations.

A field that has still undergone, in recent years, considerable developments, is the more accurate analysis of contact problems, in particular when higher-order elements are used. Such higher-order elements are typically the 10-node or 11-node tetrahedral elements generated in freeform meshing. The consistent solution algorithm proposed in ref. 28 is quite valuable in that the patch test can be satisfied exactly ${ }^{28-30}$. The algorithm is also used effectively in gluing different meshes in a multi-scale analysis (see Section 7.4), and a mathematical analysis has given insight into the performance of the discretization scheme ${ }^{30}$. 
The basic approach is that along the contactor surface, the tractions are interpolated and the gap and slip between the contactor and target are evaluated from the nodal positions and displacements. Let $\lambda$ be the contact pressure and $g(s)$ be the gap at the position $s$ along the contact surface $\Gamma_{C}$, then the normal contact conditions are

$$
g \geq 0, \quad \lambda \geq 0, \quad g \lambda=0
$$

where the last equation in (5) is the complementary condition. We use a constraint function $w_{n}(g, \lambda)$ to turn the inequality constraints of contact into the equality constraint

$$
w_{n}(g, \lambda)=0
$$

which gives in variational form

$$
\int_{\Gamma_{C}} w_{n}(g, \lambda) \delta \lambda \mathrm{d} \Gamma_{C}=0
$$

Whether the gap is open or closed on the surface is automatically contained in the formulation using the constraint function. For frictional contact conditions another constraint function is used.

The scheme published in ref. 28 should be used with appropriate interpolations for the contact pressure - for given geometry and displacement interpolations - and appropriate numerical integration schemes to evaluate the integrals enforcing the contact conditions.

The inf-sup condition for the contact discretization is given by

$$
\sup _{\mathbf{v}_{h} \in V_{h}} \frac{\int_{\Gamma_{C}} \mu_{h} g\left(\mathbf{v}_{h}\right) \mathrm{d} \Gamma_{C}}{\left\|\mathbf{v}_{h}\right\|_{1}} \geq \beta \sup _{\mathbf{v} \in V} \frac{\int_{\Gamma_{C}} \mu_{h} g(\mathbf{v}) \mathrm{d} \Gamma_{C}}{\|\mathbf{v}\|_{1}} \quad \forall \mu_{h} \in M_{h}
$$

where $M_{h}$ is the space of contact tractions and $\bullet$ is a constant, greater than zero. This condition can be satisfied as discussed in refs. 28, 30 .

For some example solutions involving contact conditions, see Sections 7.3, 7.8, 7.10, 7.11.

While we considered so far the analysis of solids and structures, of course similar considerations also hold when developing discretization schemes for fluids, including the interactions with structures, see Section 4 below, and in these cases nonlinearities are usually present.

\subsection{MEASURING THE FINITE ELEMENT SOLUTION ERRORS}

Once a finite element solution of a mathematical model has been obtained, ideally, we could assess the solution error. Much research has focused on 
the 'a posteriori' assessment of these errors ${ }^{31,32}$. However, the problem is formidable since the error between the numerical solution and the exact solution of the mathematical model shall be established when the exact solution is unknown.

A review of techniques currently available to establish this error has been published in ref. 31. It was concluded that no technique is currently available that establishes lower and upper bounds, proven to closely bracket the exact solution, when considering general analysis and an acceptable computational effort. The simple recovery-based error 'estimators' are still, in many regards, the most attractive and can of course be used in general linear and nonlinear analyses. However, they only give an indication of the error and need be used with care.

Considering recovery-based estimators, higher-order accuracy points or approximations thereof are frequently used ${ }^{33}$ but then the error estimator may only be applicable in linear analysis. In our experience, the stress bands proposed by Sussman and Bathe ${ }^{34,35}$ (see also Refs. 3 and 31) are quite effective to obtain error estimates in general analyses (see Section 7.5). Of course, in practical engineering analysis, frequently very fine meshes are used, simply to ensure that an accurate solution has been obtained. Then no error assessment is deemed necessary.

The recovery-based error estimators can be used directly to estimate the error in different regions of the domain analyzed. However, in some analyses, it may be of interest to control the error in only a specific quantity, like the bending moment at a section of the structure. Then we may need to use a fine mesh only in certain regions of the complete analysis domain. A typical example is the analysis of fluid flows with structural interactions. It may not be necessary to solve for the fluid flow very accurately in the complete fluid domain in order to only predict the stresses, accurately, in the structure at a certain location. In these cases, the concept of goal-oriented error estimation can be very effective and has considerable potential for further developments ${ }^{31,36,37}$.

\section{The Finite Element Solution of Fluids and Interactions with Structures}

The analyses of fluids and fluid structure interactions (FSI) have obtained in recent years much attention because the dynamic behavior of structures can be much influenced by surrounding fluids. If the fluid can be idealized as inviscid and undergoing only small motions, the analysis is much simpler than when actual flow and Euler or Navier-Stokes fluid assumptions are necessary. However, many analysis cases can now be modeled effectively, and when free surfaces or interactions with structures undergoing large 
deformations are considered, an arbitrary Lagrangian-Eulerian (ALE) formulation is widely used.

\subsection{ACOUSTIC FLUIDS AND INTERACTIONS WITH STRUCTURES}

The first models to describe an acoustic fluid were simply extensions of solid analysis discretizations, assuming a large bulk modulus and a small shear modulus (corresponding to the fluid viscosity). However, these fluid models are not effective because they 'lock' and even when formulated in a mixed formulation need be implemented to prevent loss of mass. In addition, the formulations then contain many zero energy modes, or modes of very small energy ${ }^{38-41}$.

A clearly more effective approach is to use a potential formulation ${ }^{3}$ (see Section 7.6) and such formulation extended also for actual flow has been used very successfully to solve large and complex fluid-structure interactions ${ }^{42}$.

\subsection{EULER AND NAVIER-STOKES FLUIDS AND FLUID STRUCTURE INTERACTIONS}

The solution of fluid flow structure interactions (FSI) requires effective finite element / finite volume techniques to model the fluid including high Péclet and Reynolds number conditions, effective finite element methods for the structure, and the proper coupling of the discretizations ${ }^{43-47}$.

Since in engineering practice, frequently rather coarse meshes are much desirable for the fluid flow (and indeed may have to be used because the 3D fluid mesh would otherwise result into too many degrees of freedom), we have concentrated our development efforts on establishing finite element discretization schemes that are stable even when coarse meshes are used for very high (element) Péclet and Reynolds numbers and show optimal accuracy $^{48-51}$. The basic approach in the development is to use flowcondition-based interpolations (FCBI) in the convective terms of the fluid and to use element control volumes (like in the finite volume method) in order to assure local mass and momentum conservation ${ }^{51-56}$.

To present the basic approach of the FCBI schemes, consider the solution of the following Navier-Stokes equations:

Find the velocity $\mathbf{v}(\mathbf{x}) \in V$ and the pressure $p(\mathbf{x}) \in P$ in the domain $\Omega$ such that

$$
\nabla \cdot \mathbf{v}=0, \quad \mathbf{x} \in \Omega
$$




$$
\begin{gathered}
\nabla \cdot(\mathbf{v} \mathbf{v}-\boldsymbol{\tau})=\mathbf{0}, \quad \mathbf{x} \in \Omega \\
\boldsymbol{\tau}=\boldsymbol{\tau}(\mathbf{v}, p)=-p \mathbf{I}+\frac{1}{\operatorname{Re}}\left\{\nabla \mathbf{v}+(\nabla \mathbf{v})^{\mathrm{T}}\right\}
\end{gathered}
$$

where we assume that the problem is well-posed in the Hilbert spaces $V$ and $P, \tau$ is the stress tensor and Re is the Reynolds number. Equations (9) and (10) are subject to appropriate boundary conditions.

In the FCBI approach, we use for the solution a Petrov-Galerkin variational formulation with subspaces $U_{h}, V_{h}$, and $W_{h}$ of $V$, and $P_{h}$ and $Q_{h}$ of $P$. The formulation for the numerical solution is:

Find $\mathbf{u}_{h} \in U_{h}, \mathbf{v}_{h} \in V_{h}$ and $p_{h} \in P_{h}$ such that for all $w \in W_{h}$ and $q \in Q_{h}$

$$
\begin{gathered}
\int_{\Omega} w \nabla \cdot\left(\mathbf{u}_{h} \mathbf{v}_{h}-\boldsymbol{\tau}\left(\mathbf{u}_{h}, p_{h}\right)\right) \mathrm{d} \Omega=\mathbf{0} \\
\int_{\Omega} q \nabla \cdot \mathbf{u}_{h} \mathrm{~d} \Omega=0
\end{gathered}
$$

The trial functions in $U_{h}$ and $P_{h}$ are the usual functions of finite element interpolations for velocity and pressure, respectively. These are selected to satisfy the inf-sup condition of incompressible analysis ${ }^{3}$. An important point is that the trial functions in $V_{h}$ are different from the functions $U_{h}$ and are defined using the flow conditions in order to stabilize the convection term. The weight functions in the spaces $W_{h}$ and $Q_{h}$ are step functions, which enforce the local conservation of momentum and mass, respectively.

The resulting FCBI elements do not require a tuning of upwind parameters, of course satisfy the property of local and global mass and momentum conservation, pass the inf-sup test of incompressible analysis and appropriate patch tests on distorted meshes, and the interpolations can be used to establish a consistent Jacobian for the iterations in the incremental step by step solutions. The elements can be used with first and second-order accuracy.

We have developed FCBI elements for incompressible, slightly compressible and low-speed compressible flows. Each of these flow categories are abundantly used in engineering practice with various turbulence models. For some solutions, see Sections 7.7, 7.8, 7.9.

In addition, of course, there is the category of high-speed compressible flows and here we use the established and widely used Roe schemes.

These fluid flow models can all be coupled with structural models, in large deformations, with contact conditions, and with piezo-electric, thermal and electro-magnetic effects ${ }^{3,47,57}$. 


\section{An Extension of the Finite Element Method - the Method of Finite Spheres}

In engineering practice, usually a major effort is necessary in a finite element analysis to establish an adequate mesh of elements. To mesh a part of a motor car may take months, when the actual computer runs, including the plotting of the results, may take only a few days.

Much research has lately been focused on the development of meshfree discretization schemes for which no mesh is used for the interpolations and numerical integrations. However, while some techniques are referred to as meshless, they are actually not truly meshless methods because a mesh is still needed for the numerical integration.

In our research we have focused on the development of the method of finite spheres (MFS) which is one of the most effective truly meshless techniques available ${ }^{58-63}$. In this technique no mesh is used, and the complete domain needs simply be covered by the spheres (or disks in 2D solutions). The nodal unknowns are located at the nodes representing the centers of the spheres. Of course, the accuracy of the numerical solution (compared to the exact solution of the mathematical model) depends on the number of spheres used. The main advantage is that the spheres (disks) overlap (whereas the classical finite elements abut to each other) and hence highly distorted or sliver elements do not exist.

The MFS can simply be understood to be a specific finite element method and, of course, the elements (disks or spheres) of the MFS can be coupled to traditional finite elements ${ }^{63}$.

So far we have tested the MFS in the analysis of solids, and have found that while the technique of course gives much more flexibility in placing nodal unknowns in the analysis, the cost of the numerical integrations is high. The traditional finite elements are much less costly in the required numerical integrations. However, further research should increase the effectiveness of the MFS and there is good potential in the technique, in particular when coupled to traditional finite elements ${ }^{63,64}$. Then the spheres would only be employed in those regions where highly distorted traditional finite elements would otherwise be used, due to the geometric complexities or the deformations that have taken place in a large deformation solution.

Of course, in principle, meshless methods can be employed also for the analysis of shells, for fluid flow simulations and FSI analyses, but the reliability issues, for example the basic phenomenon of 'locking', need be addressed as well, just like in the classical finite element discretizations ${ }^{3,59}$. 


\section{The Solution of the Algebraic Finite Element Equations}

Consider that an appropriate finite element model has been established, for a static/steady-state or transient/dynamic analysis. The next step is to solve the governing finite element equilibrium equations.

For static (steady-state) and implicit dynamic (transient) solutions, direct sparse Gauss elimination methods are effective up to model sizes of about half a million equations ${ }^{65}$. For larger models, (iterative) algebraic multi-grid methods are much more effective. For ill-conditioned systems, combinations of these techniques can be used. Since in fluid flow analyses, the number of algebraic equations to be solved is usually large, the solutions are generally obtained using an algebraic multi-grid procedure, sweeping through the momentum, continuity, turbulence, mass transfer, etc., equations.

Considering transient structural response, a widely-used scheme of time integration is the Newmark method trapezoidal rule. However, if large deformations over long-time durations need be solved, then the scheme given in ref. 66 is much more effective. In this time integration, we consider each time step $\Delta t$ to consist of two equal sub-steps, each solved implicitly. The first sub-step is solved using the trapezoidal rule with the usual assumptions

$$
\begin{aligned}
& { }^{t+\Delta t / 2} \dot{\mathbf{U}}={ }^{t} \dot{\mathbf{U}}+\left[\frac{\Delta t}{4}\right]\left({ }^{t} \ddot{\mathbf{U}}+{ }^{t+\Delta t / 2} \ddot{\mathbf{U}}\right) \\
& { }^{t+\Delta t / 2} \mathbf{U}={ }^{t} \mathbf{U}+\left[\frac{\Delta t}{4}\right]\left({ }^{t} \dot{\mathbf{U}}+{ }^{t+\Delta t / 2} \dot{\mathbf{U}}\right)
\end{aligned}
$$

Then the second sub-step is solved using the three-point Euler backward method with the governing equations

$$
\begin{aligned}
& { }^{t+\Delta t} \dot{\mathbf{U}}=c_{1}{ }^{t} \mathbf{U}+c_{2}{ }^{t+\Delta t / 2} \mathbf{U}+c_{3}{ }^{t+\Delta t} \mathbf{U} \\
& { }^{t+\Delta t} \ddot{\mathbf{U}}=c_{1}{ }_{1}^{t} \dot{\mathbf{U}}+c_{2}{ }^{t+\Delta t / 2} \dot{\mathbf{U}}+c_{3}{ }^{t+\Delta t} \dot{\mathbf{U}}
\end{aligned}
$$

where $c_{1}=1 / \Delta t, c_{2}=-4 / \Delta t, c_{3}=3 / \Delta t$.

This scheme is in essence a fully implicit second-order accurate RungeKutta method and requires per step about twice the computational effort as the trapezoidal rule. However, the accuracy per time step is significantly increased, and in particular the method remains stable when the trapezoidal rule fails to give the solution (see Section 7.12) This scheme is an option in ADINA for nonlinear dynamics, and is also employed in the ADINA FSI solutions. The Navier-Stokes and structural finite element equations are 
fully coupled in FSI solutions, and are either solved iteratively or directly using this time integration scheme.

For explicit dynamic solutions of structural response, the usual central difference method is effectively used. However, we employ this method with the same finite elements that are also used in static or implicit dynamic analyses (see Sections 7.10, 7.11). Hence the only difference to an implicit solution is that the time integrator is the central difference method and that the solution scheme can only be used with a lumped mass matrix. Of course, the time step needs to satisfy the stability limit ${ }^{3}$ and therefore many time steps are frequently used. This is clearly the method of choice for fast transient analyses.

Since the element discretizations are the same in explicit and implicit dynamic solutions, restarts from explicit to implicit solutions, and vice versa, are directly possible. This option can be of use when an initial fast transient response is followed by a slow transient, almost static response. For example, in the analysis of metal forming problems, the initial response might be well calculated using the explicit solution scheme, and the springback might be best calculated using the implicit scheme.

A key point is that explicit dynamic solutions can be obtained very efficiently using distributed memory processing (DMP) environments. The scalability is excellent, so that a hundred processors, or more, can effectively be used. This possibility renders explicit time integration very attractive, so that in some cases even solutions that normally would be obtained using implicit integration, because a relatively long time scale governs the response, might be more effectively calculated with the explicit scheme.

\section{Illustrative Solutions using ADINA}

The objective in this section is to briefly give some solutions that demonstrate the capabilities mentioned above. The solutions have all been obtained when studying the finite element methods mentioned above or using the ADINA program for the analysis of solids, structures, fluids and multi-physics problems ${ }^{67}$. Additional solutions can be found on the ADINA web site ${ }^{67}$ and, for example, in ref. 68.

\subsection{EVALUATION OF PLATE BENDING ELEMENTS}

In engineering practice mostly shell elements based on the ReissnerMindlin kinematic assumption are employed for the analysis of plates, since plates are just a special case of a shell. If a shell element (used in a plate bending analysis) is based on the Reissner-Mindlin kinematic assumption 
and satisfies the inf-sup condition for plate bending, then it does not 'lock', meaning that the convergence curves for any (admissable) plate bending problem have the optimal slope and do not shift as the thickness of the plate decreases.

Figure 1 shows a square plate, fixed around its edges. The plate is loaded with uniform pressure. Uniform meshes of the MITC4 and MITC9 shell elements have been used and the solutions were calculated for decreasing element sizes $(\mathrm{h})$ and decreasing thickness $(\mathrm{t})$ of the plate.

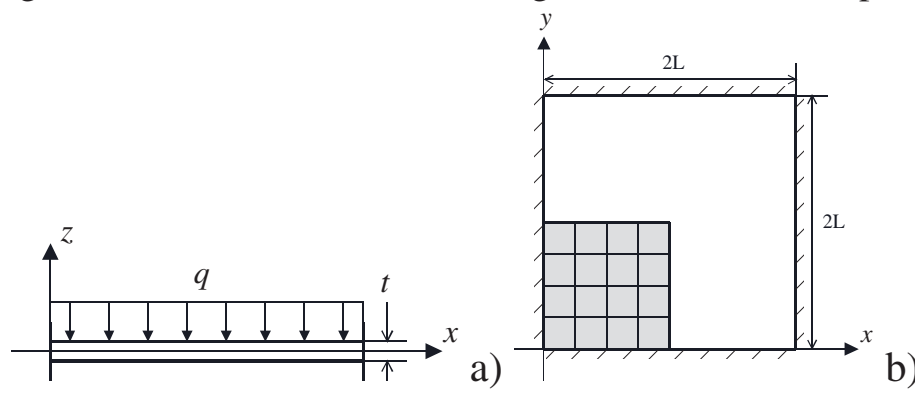

Figure 1. Clamped plate under uniform load: a) Side view of plate, b) Plan view showing also quarter of plate represented by shell elements.

Figure 2 shows the convergence curves obtained using the s-norm ${ }^{20}$. We notice that the calculated convergence curves have the optimal slope and the curves do not shift as the thickness of the plate decreases.

We show these results because it is important to test element formulations in this way - that is, the performance of a formulation in terms of convergence curves should be evaluated as the thickness of the plate decreases.

\subsection{EVALUATION OF SHELL ELEMENTS}

The thorough evaluation of shell elements is much more difficult than the evaluation of plate bending elements, because a shell element should perform well in membrane-dominated, bending-dominated and mixed problem solutions, and for any curvature shell. Hence, well-formulated linear static test problems need be selected from each of these categories, and also an appropriate norm need be used in the convergence calculations. Dynamic solutions are, of course, also of value but really only after the shell element has displayed excellent behavior in linear static solutions. 


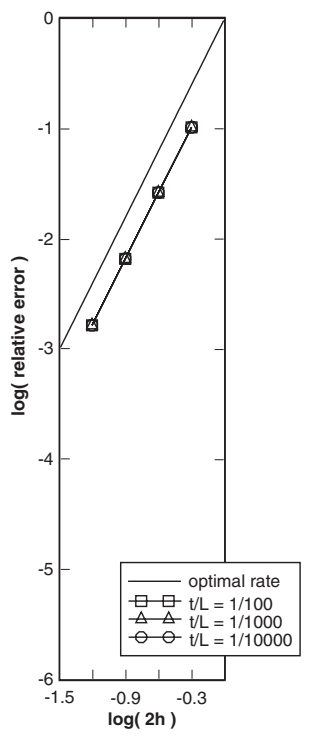

a) MITC4 element

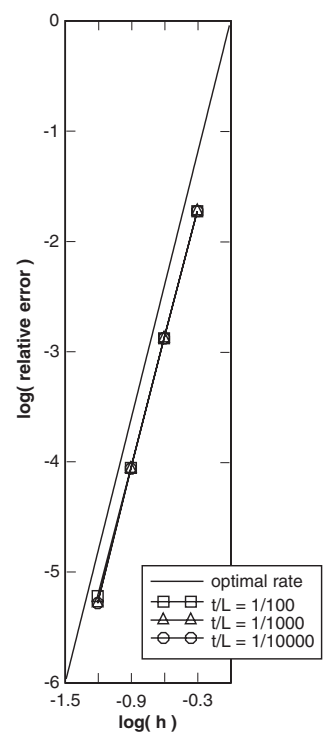

b) MITC9 element

Figure 2. Clamped plate under uniform load: convergence curves using square of the snorm.

The objective in the evaluation of a shell element must then be to identify how well the element performs in the solutions of these static analysis test problems, as the shell thickness decreases. The meshing used need to also take into account boundary layers. Ideally, in each of the test problems, the convergence curves have the optimal slope and do not shift as the shell thickness decreases.

While a number of different problems need to be solved for a full evaluation of an element, we demonstrate the task by considering the axisymmetric hyperboloid shell problem shown in Figure $3^{12}$. If the shell is clamped at both ends, the problem is membrane-dominated, and if the shell is free at both ends, the problem is bending-dominated. These are difficult test problems to solve because the shell is doubly-curved.

Figure 4 shows the convergence curves obtained when using the snorm $^{20}$. We see that the MITC4 element performs very well, independent of the shell thickness, and does not lock. 


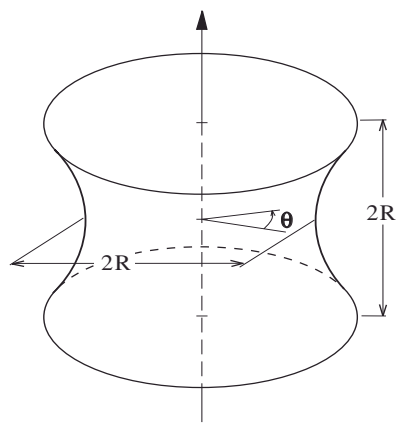

Figure 3. The axisymmetric hyperboloid shell problem. The shell is subjected to the pressure $\mathrm{p}=\mathrm{p}_{0} \cos (2 \theta)$; for the model solved using symmetry conditions see ref. 12
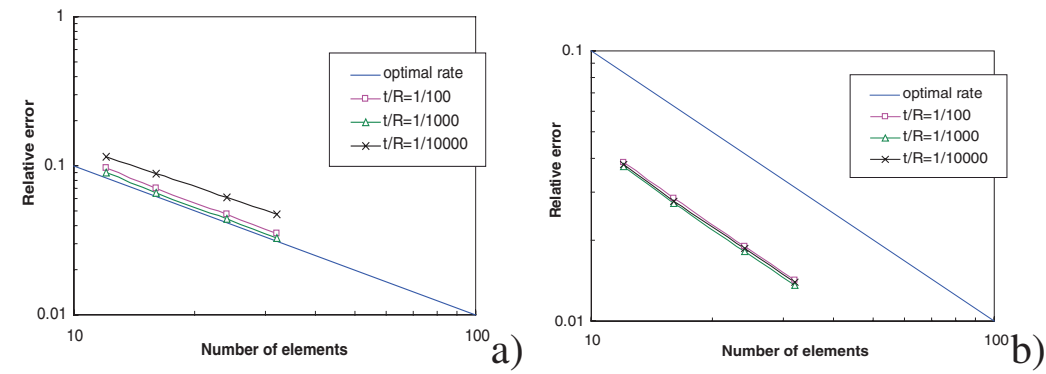

Figure 4. The axisymmetric hyperboloid shell problem: convergence curves using MITC4 element and s-norm ${ }^{12,20}$ "a) Clamped ends, b) Free ends.

\subsection{CRUSH ANALYSIS OF MOTOR CARS}

A difficult nonlinear problem involving shell analysis capabilities, multiple contact conditions, large deformations with elasto-plasticity and fracture, is the roof crush analysis of motor cars. 'Crushing' a motor car roof is a slow physical process taking seconds in contrast to 'crashing' a motor car against another object, which is an event of milliseconds.

While explicit dynamic simulations are widely used to evaluate the crash behavior of motor cars, the crushing of an automobile roof is more appropriately - and more effectively - simulated using implicit dynamic, or static, solution techniques ${ }^{65}$.

Figure 5 shows a typical finite element model of a car, and Figure 6 gives computed results for another car. The ADINA solution was obtained using an implicit dynamic (slow motion) analysis with the actual physical crushing speed of $0.022 \mathrm{mph}$. In the explicit solutions not using ADINA, quite different results were obtained for different crushing speeds, and it is seen that when the speed of crushing is close to the laboratory test speed, the explicit solution is unstable. In this explicit code, the elements used are 
not stable in static analysis (they do not satisfy the conditions mentioned in Sections 3.2 and 7.2).

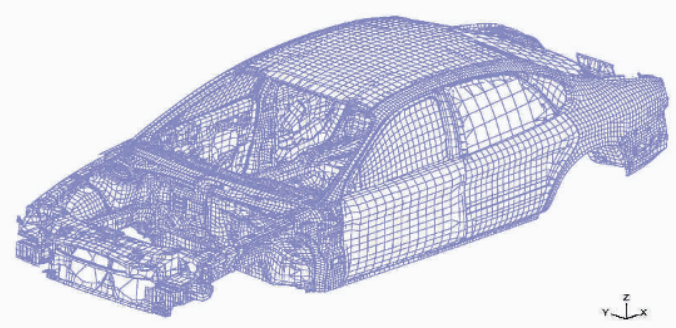

Figure 5. Finite element model of a car.

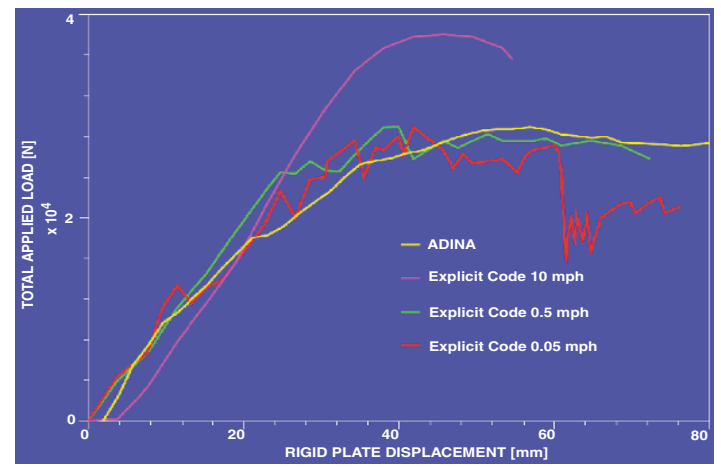

Figure 6. Results of a roof crush simulation for a car.

\subsection{GLUING OF DISSIMILAR MESHES FOR MULTI-SCALE ANALYSIS}

In a multi-scale analysis, it can be effective to create a fine mesh for a certain small region and then have successively coarser meshes away from that region. Then it may also be effective to mesh some regions with freeform tetrahedral elements while other regions are meshed with brick elements. It is often a challenge to connect these regions with dissimilar meshes together.

However, a powerful gluing feature makes it easy to connect regions with dissimilar meshes. This feature is illustrated in the simple 2D and 3D examples shown in Figures 7 and 8. The theory for this analysis feature is given in ref. 28. 


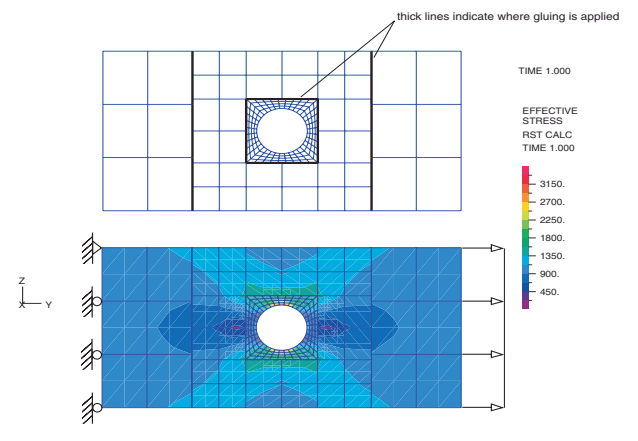

Figure 7. Gluing of dissimilar meshes, 2D.
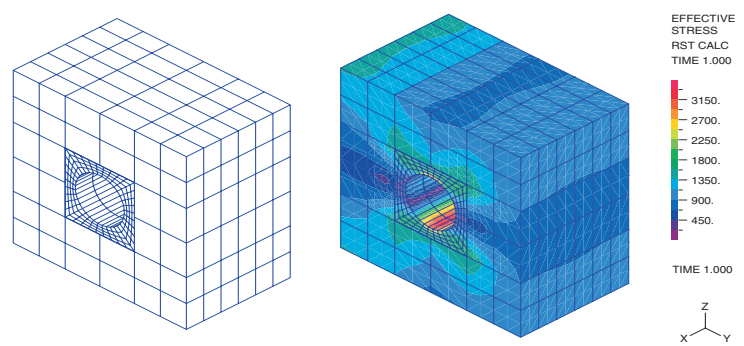

Figure 8. Gluing of dissimilar meshes, 3D.

\subsection{USING AN ERROR ESTIMATOR}

In this example, we demonstrate the use of the error estimation based on refs. 34, 35 and available in ADINA, see also ref. 31. Figures 9 and 10 show results obtained in the study of a cantilever structure with the left end fixed, in linear, nonlinear and FSI solutions. Of particular interest is the stress solution around the elliptical hole in the middle of the cantilever. In the linear analysis, the structure is simply subjected to the uniform pressure $p$. In the nonlinear analysis, this pressure is increased to $6 p$ and causes large deformations. Finally, in the fully coupled FSI solution, the steady-state force effects of a Navier-Stokes fluid flow around the structure (of magnitude about $2 p$ ) are considered in addition to the pressure of $6 p$.

In each case, a coarse mesh solution (for the mesh used see Figure 9), the error estimation for the longitudinal (bending) stress using the coarse mesh and the 'exact' error have been calculated. The exact errors have been obtained by comparing the coarse mesh solutions with very fine mesh solutions (that in practice would of course not be computed). The error estimation is seen in these cases to be conservative and not far from the exact error. 
While this error estimation can be used for general stress and thermal analyses of solids and shells, including contact conditions and FSI, a word of caution is necessary: As with all existing practical error estimation techniques, there is no proof that the error estimate is always accurate and a conservative prediction. Hence the presently available error estimation procedures are primarily useful to estimate whether the mesh is fine enough, and if a refinement is necessary where such refinement should be concentrated.

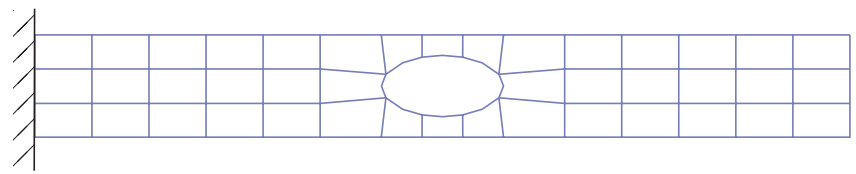

Figure 9. Coarse mesh of cantilever, used in all solutions, with boundary conditions; 9-node elements.
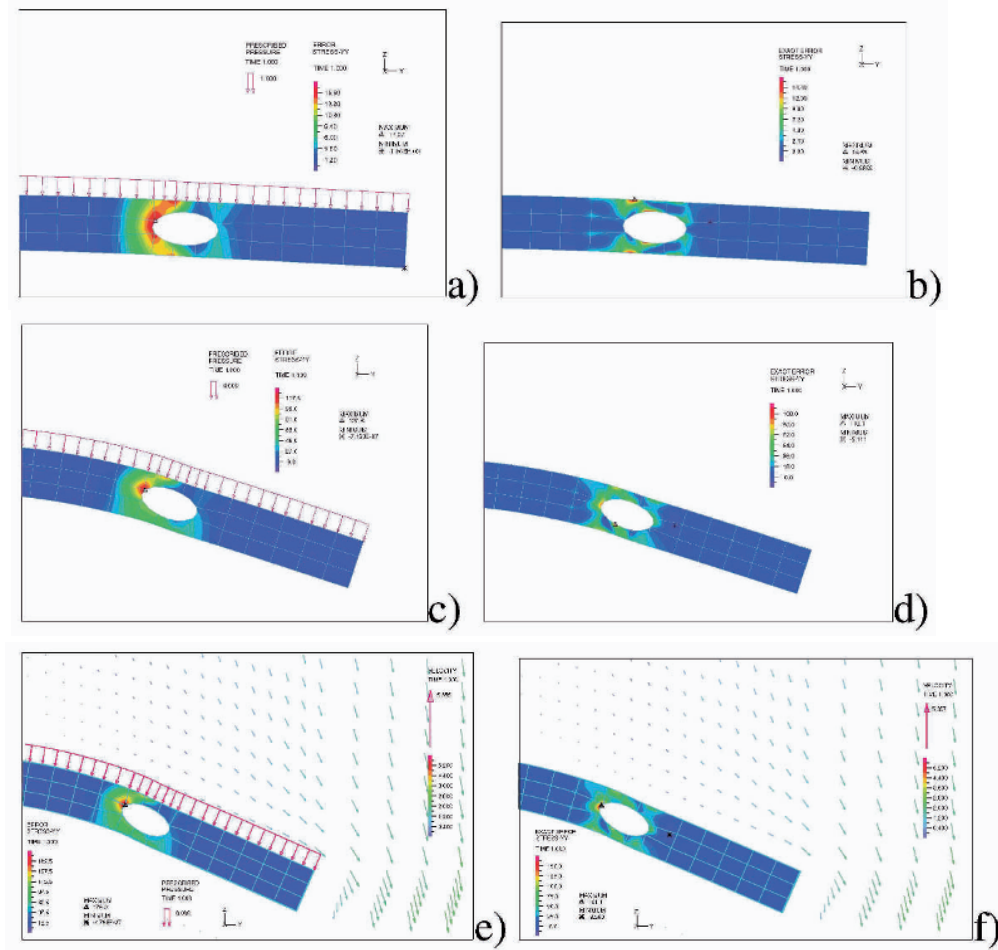

Figure 10. Results in analysis of cantilever structure: a) Linear analysis, estimated error in region of interest shown, b) Linear analysis, exact error in region of interest shown, c) Nonlinear analysis, estimated error in region of interest shown, d) Nonlinear analysis, exact error in region of interest shown, e) FSI analysis, estimated error in region of interest shown, f) FSI analysis, exact error in region of interest shown. 


\subsection{FREQUENCY SOLUTION OF FLUID STRUCTURE SYSTEM}

In many dynamic analyses of fully coupled fluid structure systems, the fluid can be assumed to be an acoustic fluid. In such cases, it can be effective to perform a frequency solution and mode superposition analysis for the dynamic response.

The major expense is then in solving for the frequencies and mode shapes, which requires the solution of the quadratic eigenvalue problem ${ }^{3}$

$$
\left(\mathbf{K}-\lambda \mathbf{C}-\lambda^{2} \mathbf{M}\right) \boldsymbol{\varphi}=\mathbf{0}
$$

The Lanczos method can be used efficiently for the solution of this eigenvalue problem.

Figures 11 and 12 show two vibration modes of a reactor vessel with piping analyzed using ADINA. The model has about 600,000 degrees of freedom and the 100 lowest frequencies and corresponding mode shapes of the quadratic eigenvalue problem were computed in about half an hour on an IBM Linux machine with four processors.

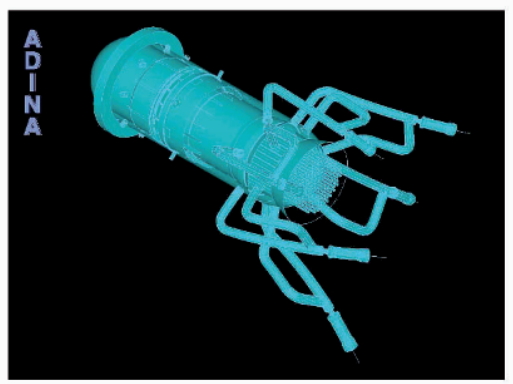

Figure 11. Low vibration mode of a reactor vessel, frequency $1.649 \mathrm{~Hz}$.

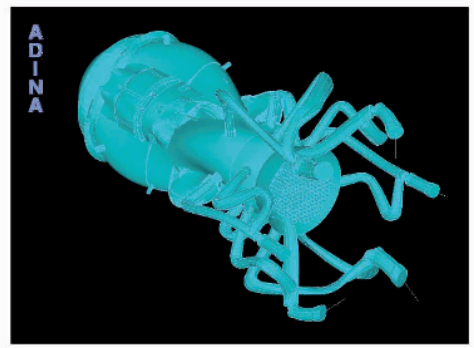

Figure 12. Higher vibration mode of a reactor vessel, frequency $39.98 \mathrm{~Hz}$. 


\subsection{SOLUTION OF LARGE FINITE ELEMENT FLUID SYSTEMS}

Today's CFD and FSI solutions require generally the solution of large finite element systems that involve millions of degrees of freedom.

Here we give two examples of solutions of large fluid flow models using an efficient algebraic multi-grid solver. Figure 13 shows the problems solved and Figure 14 gives the solution times (clock times) and memory used. It is important to note that the solution times and the memory used increase approximately linearly with the number of degrees of freedom. Also, in both problem solutions, 8 million equations are solved in less than 2 hours on the single processor PC.
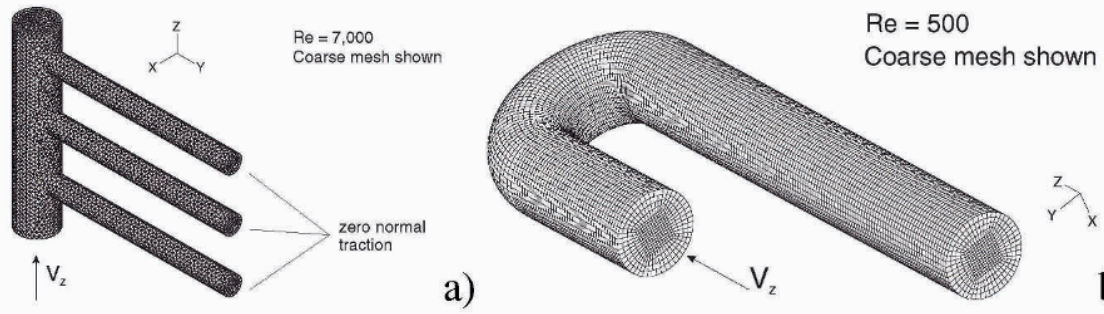

Figure 13. Models to demonstrate the solution of large finite element fluid systems: a) Manifold problem, b) Turnaround duct problem.
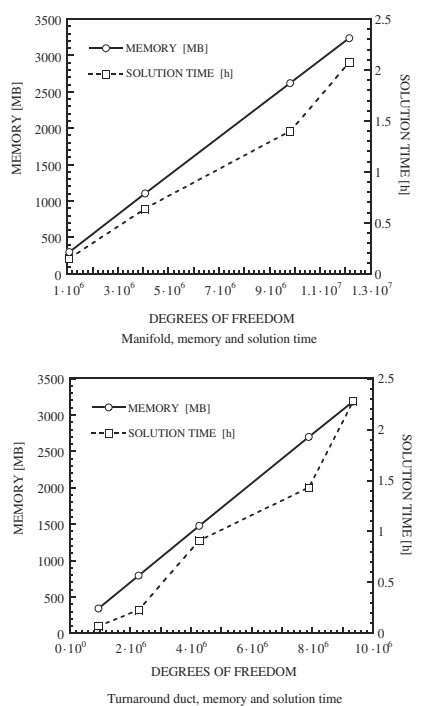

Figure 14. Solution times (clock times) and memory usage for the manifold and turnaround duct problems; single processor PC used, $3.2 \mathrm{GHz}$. 


\subsection{ANALYSIS OF OIL TANK}

There is much interest in simulating accurately the sloshing of fluids in large diameter tanks. The fluid need be modeled as a Navier-Stokes fluid and an Arbitrary-Lagrangian-Eulerian formulation is effectively used for the fully coupled FSI solution.

Figure 15 shows a typical flexible tank filled with oil. A pontoon floats on the oil surface to prevent the oil from contacting the air. The tank is subjected to a horizontal ground motion of magnitude 1 meter and frequency $0.125 \mathrm{~Hz}$.

Figure 15 also gives the ADINA model used for the analysis; here the fluid mesh ( 8 - node FCBI elements) and the structural meshes of the tank and pontoon (MITC4 shell elements) are shown separately.

Figure 16 shows some results of the analysis, namely snap-shots of the oil sloshing in the tank and stresses in the tank wall.
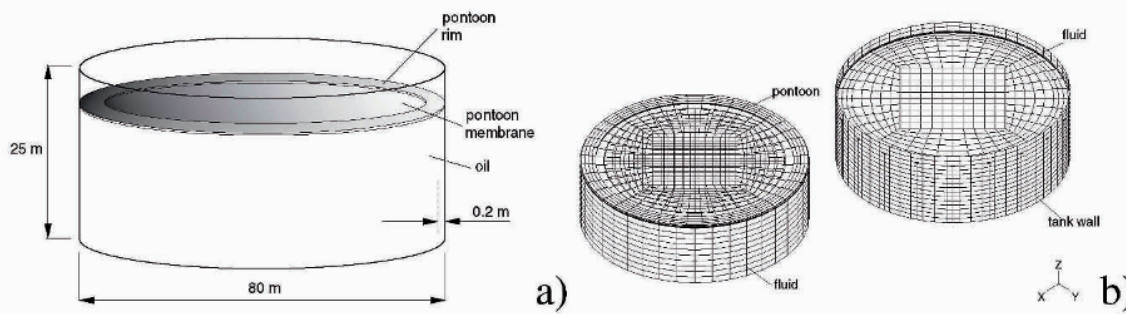

Figure 15. Analysis of oil tank: a) Schematic of tank, b) The finite element model of oil tank with pontoon.

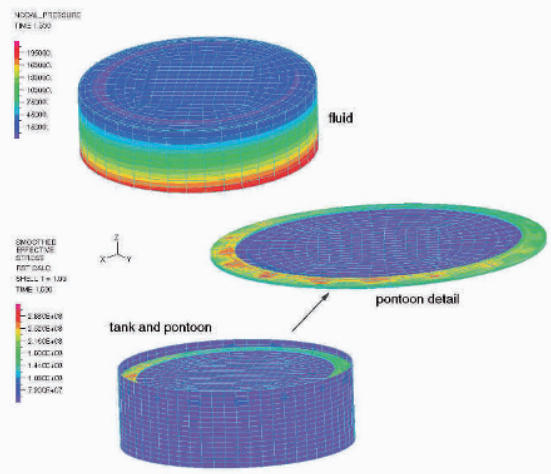

Figure 16. A snap-shot of the response of the tank. 


\subsection{ANALYSIS OF TURBULENT FLOW IN AN EXHAUST MANIFOLD}

The simulation of turbulent flow in exhaust manifolds is of much interest. Here we consider a Volvo Penta 6-cylinder diesel engine. ADINA was used with the shear stress transport (SST) turbulence model to solve for the turbulent flow in the manifold.

Figure 17 shows the manifold considered and indicates the calculated fluid flow. Figure 18 gives a detail of the manifold with the calculated pressure contours. The Reynolds number at the inlets of the manifold is approximately 13,000 , and the model was solved with about $3 \frac{1}{2}$ million unknowns. With the fluid flow also a thermo-mechanical analysis of the structure is directly possible.

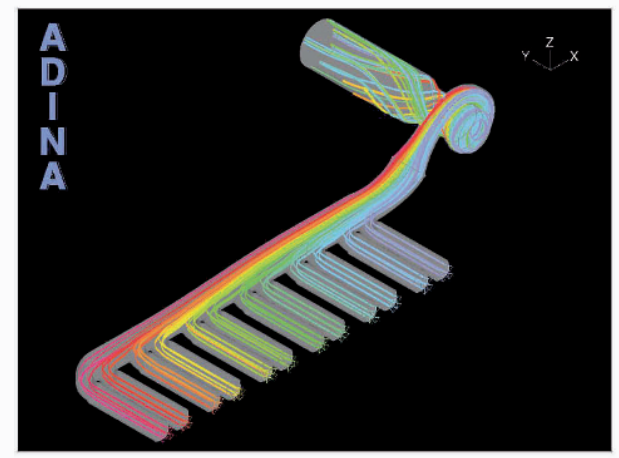

Figure 17. Exhaust manifold, also showing particle traces.

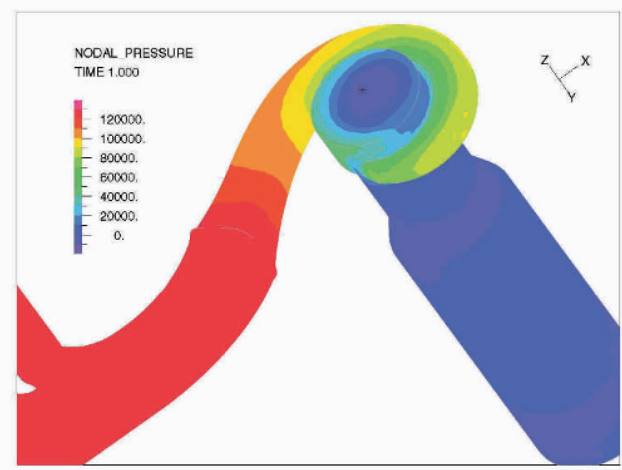

Figure 18. Exhaust manifold: pressure contours. 


\subsection{CRASH ANALYSIS OF CYLINDERS}

For very fast transient analyses, the use of explicit time integration can be much more effective than using implicit time integration. Figures 19 and 20 show the analysis of two cylinders in impact. The response has been calculated using the central difference method of explicit time integration with MITC4 shell elements used to model the cylinders. This type of analysis involving crash, contact, large deformations with fracture within milliseconds is clearly most effectively carried out using explicit time integration.

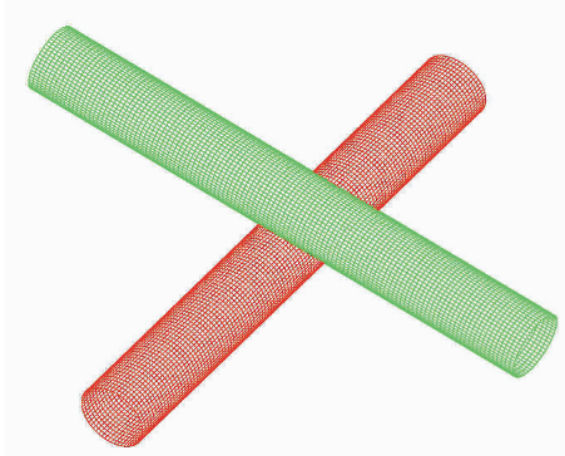

Figure 19. Crash analysis of cylinders. The top cylinder crashes onto the bottom cylinder.

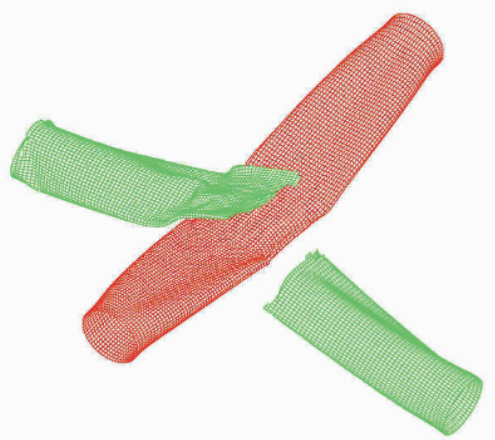

Figure 20. Response of cylinders at time $=0.040 \mathrm{~s}$.

\subsection{EXPLICIT AND IMPLICIT SOLUTIONS OF A METAL FORMING PROBLEM}

The forming of the S-rail shown in Figure 21 is a widely-used verification problem of metal forming procedures. This problem can be solved using explicit or implicit time integration in a finite element program. 


\subsection{EXPLICIT AND IMPLICIT SOLUTIONS OF A METAL FORMING PROBLEM}

The forming of the S-rail shown in Figure 21 is a widely-used verification problem of metal forming procedures. This problem can be solved using explicit or implicit time integration in a finite element program.

Figure 22 shows the results obtained using ADINA in implicit integration (the trapezoidal rule is used) and in explicit integration (the central difference method is used). The same mesh of MITC4 shell elements was employed. As seen, the final deformations and stresses are very similar using the two analysis techniques. Hence the only reason for using one or the other solution procedure in ADINA is that one technique may be computationally much more effective. In this case, the solution times are quite comparable and hence either the implicit or the explicit time integration might be used.

In this analysis ADINA was used within the NX Nastran environment and the plots were obtained using the Femap program.

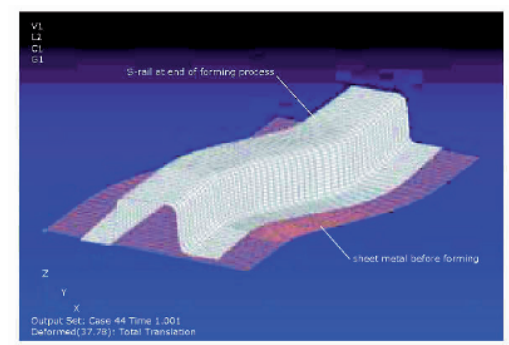

Figure 21 Forming of an S-rail.
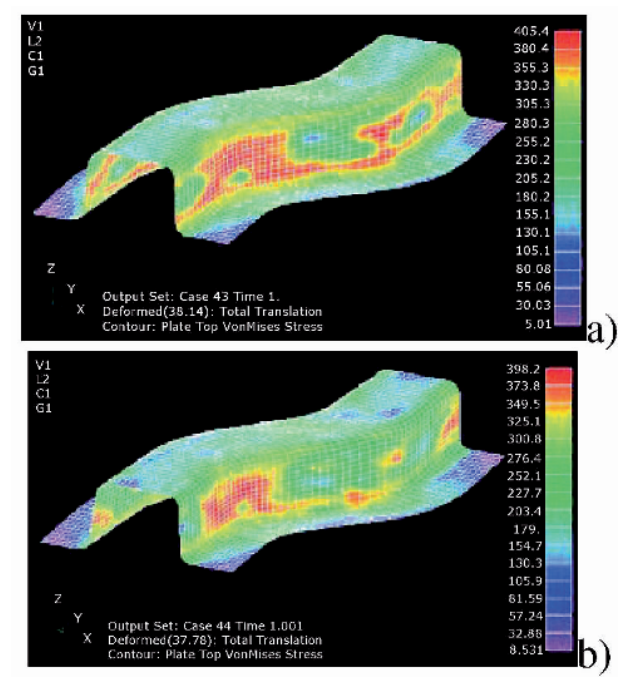

Figure 22. Results obtained in forming of the S-rail: a) Results using implicit integration, b) Results using explicit integration. 


\subsection{THE NONLINEAR DYNAMIC LONG DURATION SOLUTION OF A ROTATING PLATE}

The Newmark method trapezoidal rule of time integration can become unstable in large deformation, long time duration analyses, and in such cases the time integration scheme given in equations (14) to (17) can be much more effective.

Figure 23 shows a plate, free to rotate, which is subjected to a twisting moment. Once the moment is removed, the plate should continue to rotate at constant angular speed.

Figure 24 gives the kinetic energy of the plate as a function of time. If the trapezoidal rule of time integration is used, the solution becomes unstable after a few rotations, although Newton-Raphson equilibrium iterations to a tight convergence tolerance are performed in each time step. On the other hand, the scheme of equations (14) to (17) with a much larger time step gives a stable and accurate solution.

More experiences with this time integration scheme are given in ref. 66.

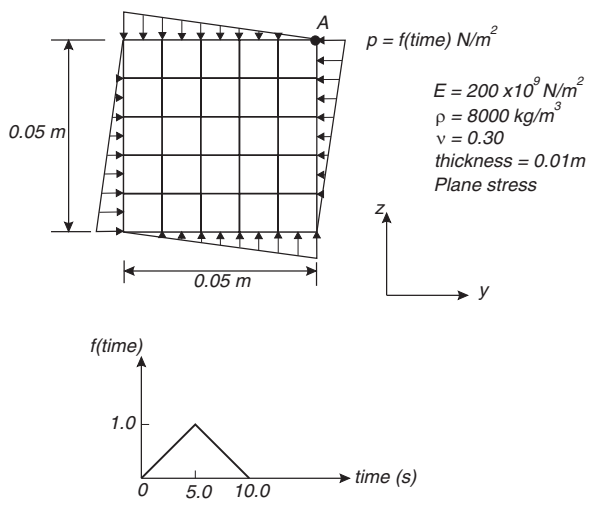

Figure 23. Rotating plate problem

\section{Concluding Remarks}

The objective in this paper was to present some developments regarding the analysis of structures when subjected to severe loading conditions. The required simulations will frequently result in highly nonlinear analyses, involving multi-physics conditions with fluid-structure interactions and thermal effects.

The focus in this paper was on the need to use reliable finite element methods for such simulations. Since test data for the envisioned scenarios will be scarce, it is important to have as high a confidence as possible in the computed results without much experimental verification. Such confidence 
is, however, only possible if reliable finite element methods are used. Furthermore, it can be effective to employ a single program system to perform the analyses, in hierarchical modeling involving linear to highly nonlinear solutions.

In this paper various solutions obtained with ADINA have been presented that show the applicability and versatility of the program in the study of linear and highly nonlinear problems, including multi-physics conditions.

While many different and complex problems can at present be solved, there are still major challenges in developing more effective and more comprehensive analysis procedures. Eight key challenges have been summarized in ref. 1, see the Preface of the 2005 Volume.
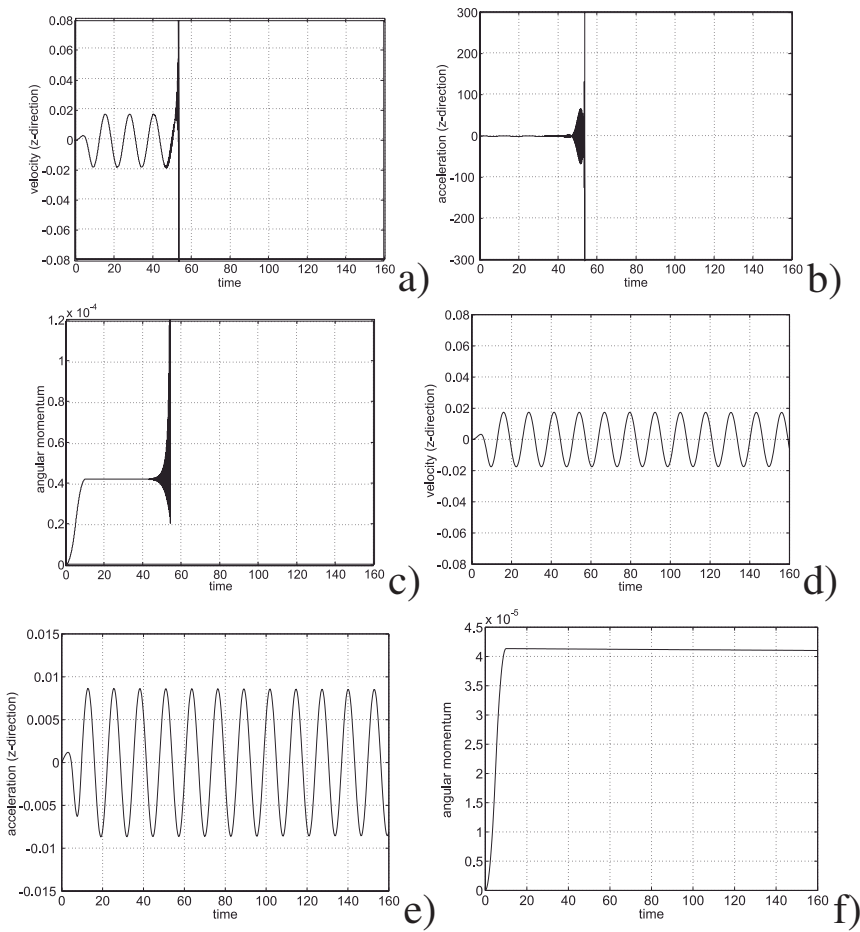

Figure 24. Results obtained in the analysis of the rotating plate problem: a) Velocity at point A using the trapezoidal rule; $\Delta \mathrm{t}=0.02 \mathrm{~s}$, b) Acceleration at point A using the trapezoidal rule; $\Delta \mathrm{t}=0.02 \mathrm{~s}, \mathrm{c})$ Angular momentum using the trapezoidal rule; $\Delta \mathrm{t}=0.02 \mathrm{~s}$, d) Velocity at point A using the scheme of Equations (14) to (17); $\Delta \mathrm{t}=0.4 \mathrm{~s}$, e) Acceleration at point $\mathrm{A}$ using the scheme of Equations (14) to (17); $\Delta \mathrm{t}=0.4 \mathrm{~s}, \mathrm{f}$ ) Angular momentum using the scheme of Equations (14) to (17); $\Delta \mathrm{t}=0.4 \mathrm{~s}$ 


\section{References}

1. K. J. Bathe, (ed.), Computational Fluid and Solid Mechanics, (Elsevier, 2001); Computational Fluid and Solid Mechanics 2003, (Elsevier, 2003); Computational Fluid and Solid Mechanics 2005, (Elsevier, 2005). Proceedings of the First to Third MIT Conferences on Computational Fluid and Solid Mechanics.

2. O.C. Zienkiewicz and R.L. Taylor, The Finite Element Method, (ButterworthHeinemann, 2005).

3. K. J. Bathe, Finite Element Procedures, (Prentice Hall, 1996).

4. M. L. Bucalem and K. J. Bathe, The Mechanics of Solids and Structures - Hierarchical Modeling and the Finite Element Solution, (Springer, to appear).

5 K. J. Bathe, The inf-sup condition and its evaluation for mixed finite element methods, Computers \& Structures, 79:243-252, 971, (2001).

6. F. Brezzi and M. Fortin, Mixed and Hybrid Finite Element Methods, Springer Verlag, New York, 1991.

7. D. Pantuso and K. J. Bathe, A four-node quadrilateral mixed-interpolated element for solids and fluids, Mathematical Models and Methods in Applied Sciences, 5(8):11131128, (1995).

8. D. Pantuso and K. J. Bathe, On the stability of mixed finite elements in large strain analysis of incompressible solids, Finite Elements in Analysis and Design, 28:83-104, (1997).

9. A. Iosilevich, K. J. Bathe and F. Brezzi, Numerical inf-sup analysis of MITC plate bending elements, Proceedings, 1996 American Mathematical Society Seminar on Plates and Shells, Québec, Canada, (1996).

10. A. Iosilevich, K. J. Bathe and F. Brezzi, On evaluating the inf-sup condition for plate bending elements, Int. Journal for Numerical Methods in Engineering, 40:3639-3663, (1997).

11. K. J. Bathe, A. Iosilevich and D. Chapelle, An inf-sup test for shell finite elements, Computers \& Structures, 75: 439-456, (2000).

12. D. Chapelle and K.J. Bathe, The Finite Element Analysis of Shells - Fundamentals, (Springer, 2003).

13. D. Chapelle and K. J. Bathe, Fundamental considerations for the finite element analysis of shell structures, Computers \& Structures, 66(1):19-36, (1998).

14. K. J. Bathe, A. Iosilevich and D. Chapelle, An evaluation of the MITC shell elements, Computers \& Structures, 75: 1-30, (2000).

15. M. L. Bucalem and K. J. Bathe, Finite element analysis of shell structures, Archives of Computational Methods in Engineering, 4:3-61, (1997).

16. K. J. Bathe, P. S. Lee and J. F. Hiller, Towards improving the MITC9 shell element, Computers \& Structures, 81: 477-489, (2003).

17. P. S. Lee and K. J. Bathe, Development of MITC isotropic triangular shell finite elements, Computers \& Structures, 82:945-962, (2004).

18. P. S. Lee and K. J. Bathe, On the asymptotic behavior of shell structures and the evaluation in finite element solutions, Computers \& Structures, 80:235-255, (2002).

19. K. J. Bathe, D. Chapelle and P. S. Lee, A shell problem 'highly-sensitive' to thickness changes, Int. Journal for Numerical Methods in Engineering, 57:1039-1052, (2003).

20. J. F. Hiller and K. J. Bathe, Measuring convergence of mixed finite element discretizations: an application to shell structures, Computers \& Structures, 81:639-654, (2003).

21. D. Chapelle and K. J. Bathe, The mathematical shell model underlying general shell elements, Int. J. for Numerical Methods in Engineering, 48:289-313, (2000). 
P. S. Lee and K. J. Bathe, Insight into finite element shell discretizations by use of the basic shell mathematical model, Computers \& Structures, 83:69-90, (2005).

23. D. Chapelle, A. Ferent and K. J. Bathe, 3D-shell elements and their underlying mathematical model, Mathematical Models \& Methods in Applied Sciences, 14:105-142, (2004).

24. M. Kojic and K.J. Bathe, Inelastic Analysis of Solids and Structures, (Springer, 2005).

25. G. Gabriel and K. J. Bathe, Some computational issues in large strain elasto-plastic analysis, Computers \& Structures, 56(2/3):249-267, (1995).

26. K. J. Bathe and F. J. Montans, On modeling mixed hardening in computational plasticity, Computers \& Structures, 82:535-539, (2004).

27. F. J. Montans and K. J. Bathe, Computational issues in large strain elasto-plasticity: an algorithm for mixed hardening and plastic spin, Int. J. for Numerical Methods in Eng., 63:159-196, (2005).

28. N. Elabbasi and K. J. Bathe, Stability and patch test performance of contact discretizations and a new solution algorithm, Computers \& Structures, 79:1473-1486, (2001).

29. N. Elabbasi, J. W. Hong and K. J. Bathe, The reliable solution of contact problems in engineering design, Int. J. of Mechanics and Materials in Design, 1:3-16, (2004).

30. K. J. Bathe and F. Brezzi, Stability of finite element mixed interpolations for contact problems, Proceedings della Accademia Nazionale dei Lincei, s. 9, 12:159-166, (2001).

31 T. Grätsch and K. J. Bathe, A posteriori error estimation techniques in practical finite element analysis, Computers \& Structures, 83:235-265, (2005).

32 M. Ainsworth and J.T. Oden, A posteriori Error Estimation in Finite Element Analysis, J. Wiley \& Sons, 2000.

33. J. F. Hiller and K. J. Bathe, Higher-order-accuracy points in isoparametric finite element analysis and an application to error assessment, Computers \& Structures, 79:1275-1285, (2001).

34. T. Sussman and K. J. Bathe, Studies of Finite Element Procedures - On Mesh Selection, Computers \& Structures, Vol. 21, pp. 257-264, 1985.

35. T. Sussman and K. J. Bathe, Studies of Finite Element Procedures - Stress Band Plots and the Evaluation of Finite Element Meshes, Engineering Computations, Vol. 3, pp. 178-191, 1986

36. T. Grätsch and K. J. Bathe, Influence functions and goal-oriented error estimation for finite element analysis of shell structures, Int. J. for Numerical Methods in Eng., 63:709-736, (2005).

37. T. Grätsch and K. J. Bathe, Goal-oriented error estimation in the analysis of fluid flows with structural interactions, Comp. Meth. in Applied Mech. and Eng., in press.

38. K. J. Bathe, C. Nitikitpaiboon and X. Wang, A mixed displacement-based finite element formulation for acoustic fluid-structure interaction, Computers \& Structures, 56:(2/3), 225-237, (1995).

39. X. Wang and K. J. Bathe, On mixed elements for acoustic fluid-structure interactions, Mathematical Models \& Methods in Applied Sciences, 7(3):329-343, (1997).

40. X. Wang and K. J. Bathe, Displacement/pressure based mixed finite element formulations for acoustic fluid-structure interaction problems, Int. Journal for Numerical Methods in Engineering, 40:2001-2017, (1997).

41. W. Bao, X. Wang, and K. J. Bathe, On the inf-sup condition of mixed finite element formulations for acoustic fluids, Mathematical Models \& Methods in Applied Sciences, 11(5):883-901, (2001).

42. T. Sussman and J. Sundqvist, Fluid-structure interaction analysis with a subsonic potential-based fluid formulation, Computers \& Structures, 81:949-962, (2003). 
43. K. J. Bathe, Simulation of structural and fluid flow response in engineering practice, Computer Modeling and Simulation in Engineering, 1:47-77, (1996).

44. K. J. Bathe, H. Zhang and S. Ji, Finite element analysis of fluid flows fully coupled with structural interactions, Computers \& Structures, 72:1-16, (1999).

45. S. Rugonyi and K. J. Bathe, On the finite element analysis of fluid flows fully coupled with structural interactions, Computer Modeling in Engineering \& Sciences, 2:195-212, (2001).

46. S. Rugonyi and K. J. Bathe, An evaluation of the Lyapunov characteristic exponent of chaotic continuous systems, Int. Journal for Numerical Methods in Engineering, 56:145163, (2003).

47. K. J. Bathe and H. Zhang, Finite element developments for general fluid flows with structural interactions, Int. Journal for Numerical Methods in Engineering, 60:213-232, (2004).

48. K. J. Bathe, D. Hendriana, F. Brezzi and G. Sangalli, Inf-sup testing of upwind methods, Int. J. for Numerical Methods in Engineering, 48:745-760, (2000).

49. Y. Guo and K. J. Bathe, A numerical study of a natural convection flow in a cavity, Int. J. for Numerical Methods in Fluids, 40:1045-1057, (2002).

50. D. Hendriana and K. J. Bathe, On upwind methods for parabolic finite elements in incompressible flows, Int. J. for Numerical Methods in Engineering, 47:317-340, (2000).

51. K. J. Bathe and H. Zhang, A Flow-Condition-Based Interpolation finite element procedure for incompressible fluid flows, Computers \& Structures, 80:1267-1277, (2002).

52. K. J. Bathe and J. P. Pontaza, A Flow-Condition-Based Interpolation mixed finite element procedure for high Reynolds number fluid flows, Mathematical Models and Methods in Applied Sciences, 12(4): 525-539, (2002).

53. H. Kohno and K. J. Bathe, Insight into the Flow-Condition-Based Interpolation finite element approach: Solution of steady-state advection-diffusion problems, Int. J. for Numerical Methods in Eng., 63:197-217, (2005).

54. H. Kohno and K. J. Bathe, A Flow-Condition-Based Interpolation finite element procedure for triangular grids, Int. J. Num. Meth. in Fluids, 49:849-875, (2005).

55. H. Kohno and K. J. Bathe, A 9-node quadrilateral FCBI element for incompressible Navier-Stokes flows, Comm. in Num. Methods in Eng., (in press).

56. B. Banijamali and K. J. Bathe, The CIP Method embedded in finite element discretizations of incompressible fluid flows, submitted.

57. P. Gaudenzi and K. J. Bathe, An iterative finite element procedure for the analysis of piezoelectric continua, J. of Intelligent Material Systems and Structures, 6(2): 266-273, (1995).

58. S. De and K. J. Bathe, The method of finite spheres, Computational Mechanics, 25:329$345,(2000)$.

59. S. De and K. J. Bathe, Displacement/pressure mixed interpolation in the method of finite spheres, Int. J. for Numerical Methods in Engineering, 51:275-292, (2001).

60. S. De and K. J. Bathe, Towards an efficient meshless computational technique: the method of finite spheres, Engineering Computations, 18:170-192, (2001).

61. S. De and K. J. Bathe, The method of finite spheres with improved numerical integration, Computers \& Structures, 79:2183-2196, (2001).

62. S. De, J. W. Hong and K. J. Bathe, On the method of finite spheres in applications: Towards the use with ADINA and in a surgical simulator, Computational Mechanics, 31:27-37, (2003). 
63. J. W. Hong and K. J. Bathe, Coupling and enrichment schemes for finite element and finite sphere discretizations, Computers \& Structures, 83:1386-1395, (2005).

64. M. Macri and S. De, Towards an automatic discretization scheme for the method of finite spheres and its coupling with the finite element method, Computers \& Structures, 83:1429-1447, (2005).

65. K. J. Bathe, J. Walczak, O. Guillermin, P. A. Bouzinov and H. Chen, Advances in crush analysis, Computers \& Structures, 72:31-47, (1999).

K. J. Bathe and M. M. I. Baig, On a composite implicit time integration procedure for nonlinear dynamics, Computers \& Structures, 83:2513 - 2534, (2005).

67. K. J. Bathe, ADINA System, Encyclopaedia of Mathematics, 11:33-35, (1997); http://www.adina.com.

68 J. Tedesco and J. Walczak, Guest Editors of Special Issue, Computers and Structures, 81:455-1109,(2003). 